\title{
A mHealth-intervention to support the self-management of multimorbid patients in general practices in Germany (TelePraCMan) - Study protocol of a randomized pilot study
}

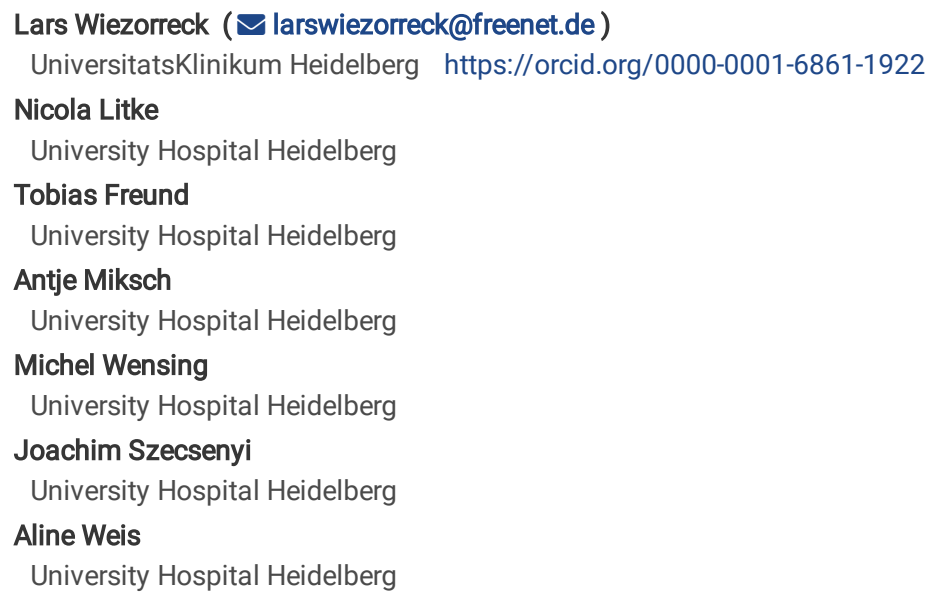




\section{Abstract}

Background: Previously we developed a structured case management for patients with multiple chronic conditions in primary care (called 'PraCMan'), delivered by Health Care Assistants in General Practice (VERAHs). The 'TelePraCMan' project aims to develop a supplementary web-based application to 'PraCMan', including a digital symptom diary in which patients can enter individual data via smartphone/tablet. This application is targeted at patients suffering from diabetes mellitus type 2, COPD, hypertension and/or chronic heart failure.

Objective: The objective of this study is to evaluate this mHealth-intervention for patients with chronic diseases, focused on patients' quality of life and selfmanagement capabilities.

Methods: A two-armed patient-randomized pilot trial is planned, involving 200 multimorbid patients in 20 GP-practices that use 'PraCMan' regularly. Participants are randomly assigned (1:1) to receive either standard 'PracMan' treatment or standard 'PracMan' treatment complemented with the mHealthintervention (TelePraCMan). As primary outcome, the health-related quality of life is measured by the German version of the Short Form Health Questionnaire (SF-12). Secondary outcomes include self-management capabilities, technical affinity, and user experience of patient and practice staff. Outcomes are measured at baseline (T0) and at follow-up after 6 months (T1). For the evaluation of the implementation process, GPs, VERAHs and patients will be invited to participate in semi structured interviews afterwards.

The outcomes will be compared between study arms using multilevel regression analysis. Qualitative interviews will be transcribed and analysed using qualitative content analysis based on the Tailored Implementation for Chronic Diseases Framework.

Discussion: 'TelePraCMan' is one of the first mHealth-Interventions addressing multimorbid patients in primary care.

Trial registration: DRKS, DRKS00017320, Registered 18 June 2019, http://www.drks.de/DRKS00017320

\section{Background}

The demographic change is associated with an increasing number of chronically ill patients leading to a rising need for care, especially in ambulatory care [1, 2]. The presence of several chronic diseases of equal importance in a person [3] is more likely in individuals of higher and advanced age [4, 5]. This poses challenges for primary care, which manages most of these patients and suffers from shortages of general practitioners (GP) [6]. To address this, innovative care models are required. One of these innovative models is the 'PraCMan'-program, which became part of the everyday care within the German federal state of Baden-Wuerttemberg in 2014 to support patients suffering from multiple chronic diseases [7].

'PraCMan' is a model for structured management of chronic diseases in primary care. Patients in 'PraCMan' not only receive regular software-supported monitoring by a care assistant in the general practice (Versorgungsassistentin in der Hausarztpraxis/ VERAH), but are also encouraged to actively participate in the management of their disease(s) by, for example, keeping paper-based symptom diaries for chronic obstructive pulmonary disease (COPD), type 2 diabetes mellitus, hypertension and chronic heart failure. However, the uptake of self-management support in general practices is suboptimal. For instance, the diaries are not handed out to all patients and, if handed out, they often appear to be filled out inaccurately. As a result, the offered symptom diaries are hardly used by patients and GPs. This is consistent with other research in chronically ill patients, which showed that 35-43\% of patients do not use paper-based symptom diaries handed out by their GPs [8,9]. Nevertheless, symptom diaries are described as an important source of information for the attending physician and can enable the patient to cope with his illnesses in a reflective manner as an important prerequisite for self-management [10]. Observation of vital signs and symptoms by multimorbid patients is an important aspect to maintain health-related quality of life [11] as multimorbidity is characterized by a strong interaction between physical impairments and the health-related quality of life [11, 12].

Mobile health technologies (mHealth) such as smartphones, mobile applications and wearable devices have shown to offer significant and innovative opportunities for improving patient to provider communication and self-management of single chronic conditions, such as diabetes [13]. In the research literature, we identified only one study on a mHealth-intervention that explicitly addressed patients with multiple chronic diseases in the primary care setting [14-19]. This study showed that multimorbid patients accept and use mHealth-interventions. Furthermore, the intervention was able to support them in selfmanagement $[18,20]$. Nevertheless, there arose challenges of successfully integrating the intervention into the patients' everyday life as well as into the workflows of the GP-practices $[18,19]$. Significant changes in patient outcomes referring to quality of life and activation could not be shown in a four-months trail [18]. This was traced back to the small sample of the study.

Furthermore, few studies explored the acceptance and use of mHealth-interventions by older peoples in Germany generally [20-23]. Studies of acceptance and effectiveness of mHealth-interventions for multimorbid patients and older people show that further research is needed to better adapt mHealthinterventions and enhance their application in routine healthcare practice.

The project 'TelePraCMan' aims to support patients' self-management capabilities and health-related quality of life by introducing a mHealth-intervention for monitoring in the context of 'PraCMan', an established model of structured primary care for patients with multimorbidity.

\section{Objectives}

\section{Primary objective}

The primary objective of this study is to determine whether the use of 'TelePraCMan' as an addition to the GP-practice-based care management intervention (PraCMan) has an influence on patients' health-related quality of life as compared to regular treatment via 'PraCMan' [24, 25].

Loading [MathJax]/jax/output/CommonHTML/jax.js 


\section{Secondary objectives}

We will explore the self-management capabilities and need of technical support of patients as well as GPs and VERAHs acceptance of the application. Furthermore, general technical affinity of patients, GPs and VERAHs as well as user experiences referring to 'TelePraCMan' by patients will be explored. Also, we will investigate whether the required technologies (Smartphone/Tablet) are available within the target group of multimorbid 'PraCMan'-patients Conclusively, the general feasibility and implementation of 'TelePraCMan' into the care system will be explored.

The hypothetical working mechanisms of 'TelePraCMan' are shown in Figure 1

\section{Methods}

This study protocol was structured based on the SPIRIT 2013 guidelines [26].

\section{Study design}

The evaluation is designed as a parallel-group, patient-randomized, multi-center pilot trial in GP-practices in the federal state of Baden-Wuerttemberg, Germany (fig. 2). Patients will be randomized to the intervention and control group to be equally sized (1:1). The intervention group will use the application for six months, the control group will continuously be treated by the standard PraCMan treatment using paper-based symptom protocols. Blinding of either patients or GP-practice teams was not possible due to the character of intervention.

All participating patients as well as GPs and VERAHs in the participating practices take part in the baseline measurement (T0). Patients assigned to the intervention group receive access to 'TelePraCMan' within the randomization process and will be using 'TelePraCMan' for six months. After at least six months, all participants (patients, GPs and VERAHs) will receive a follow-up questionnaire (T1). Furthermore, the patients as well as VERAHs and GPs receive an invitation to report their experiences with 'TelePraCMan' in additional interviews within the process evaluation.

\section{Study setting}

The study is being conducted in GP-practices in the federal state of Baden-Wuerttemberg in the south-west of the Federal Republic of Germany. The Department of General Practice and Health Services Research at University Hospital Heidelberg carries out the study and takes the role of the study central office.

\section{Eligibility criteria}

'TelePraCMan' addresses multimorbid patients who are enrolled in the case management program 'PraCMan'. Within this study we refer to the most common definition of multimorbidity, which sees multimorbidity as the presence of two or more diseases $[27,28]$. Therefore, patients who are suffering from at least two of the following conditions: diabetes mellitus type 2,COPD, high blood pressure and/or heart failure will be included in this study.

Practices eligible for study participation have to be part of the 'PraCMan' program for a minimum of six months, covering at least 20 'PraCMan' patients.

For their participation, patients need access to an internet-ready portable device. Furthermore, patients need to be aged 18 years or older and be able to read and speak German and to give consent for study participation. Because both, patients of the control group and of the intervention group, are included in 'PraCMan', the basic quality of care remains the same. For the inclusion in 'PraCMan', patients must be insured at the sickness fund 'AOK-Baden-Wuerttemberg' and have to be enrolled in the general practice-centered care contract [29].

Patients who do not fit the named eligibility criteria will not be included in this study. The technical affinity of the patients will not influence the inclusion in this study. The requested portable devices may also belong to patients' relatives who are welcome to help the patients fill in their digital symptom diaries.

\section{Intervention}

\section{'TelePraCMan' development}

'TelePraCMan' was developed and programmed by the Department of General Practice and Health Services Research at the University Hospital Heidelberg. The initial objective of developing 'TelePraCMan' was to provide the various paper-based symptom diaries of the primary care case-management program PraCMan'digitally $\in$ anappf or smartpho $\neq$ or $t a b \leq t$. In'PraCMan', thepatientshodkeepthesesymp $\rightarrow$ mdiariesbetweenregarmoni $\rightarrow r \in$ TelePraCMan' besides the initial basic symptom diaries. Furthermore, 'TelePraCMan' has been transformed into a web-based application. This means that the application is not installed locally on the user's smartphone or tablet. Instead, data processing takes place on independent web servers (of University Hospital Heidelberg) and access is realized via a web browser on the user's smartphone or tablet which means that an internet connection is required in order to use the app.

\section{'TelePraCMan' features}

'TelePraCMan' has four main features: 1) Symptom diaries for diabetes mellitus type 2, blood pressure, heart failure, and COPD, 2) an appointment manager, and 3) a manager for target agreements, which are made individually for each patient within the regular 'PraCMan' monitoring in collaboration with GP and VERAH. The symptom diaries were supplemented by 4) a graphic progress display of vital signs and symptoms that have been entered.ain functions of 
The patient is expected to actively enter his or her data in 'TelePraCMan'. An automatic data transmission, e.g. from the blood glucose meter is not provided. By dealing with the data, the patient should gain better awareness of the vital signs and symptoms and the influence his behaviour can have on them [30]. A reminder can be viewed on ballot screen of 'TelePraCMan' by user.

\section{Implementation strategies}

To achieve adequate participant enrolment, we financially incentivized participation of GP-practice teams and patients. Practice teams will receive a short training imparting knowledge on the use of 'TelePraCMan' and the remote access for GP-practices. Every intervention group participant also receives a step-bystep instruction manual and a configuration checklist from his GP-practice. The configuration of symptom diaries (selection of diaries, limit and target values, diary rhythm) and target agreements are individually negotiated by patient, VERAH and GP. The patient then configures 'TelePraCMan' using the configuration checklist, which contains the negotiated settings.

\section{Outcome measures}

\section{Primary outcome}

Effects on the patients' health related quality of life during the 6-months intervention period will be assessed using the German version of the Short Form 12 Health Questionnaire (SF-12 [31]). This outcome will be collected at baseline at the beginning of the intervention (T0) and after six months at the end of the intervention (T1).

\section{Secondary outcome}

Secondary outcomes for participation patients are defined as follows:

- The patients' self-management capabilities is measured using the German version of the "Patient Activation Measure" (PAM13-D [32]). Self-management is the hypothesized moderating variable between usage of 'TelePraCMan' and health-related quality of life according to the rationale that selfmanagement respectively empowerment results into a better health-related quality of life. Studies showed an association between higher patient activation and a better health related quality of life [32-34].

- A questionnaire to general technical affinity (TA-EG [35]) of participating patients and a smartphone usage questionnaire (self-administered) aim to measure the general openness towards and acceptance of new or digital technology like mHealth-interventions. The self-administered smartphone usage questionnaire is a 13-items instrument that collects ownership (yes/no), purchase (self-bought/ receive as present/ other), and frequency of use in everyday life as well as one item set about frequency of use of various smartphone functions (5-point Likert scale with response options from 'never' to 'very often').

- The objective number and frequency of use of 'TelePraCMan' by patients is determined by log-in data of users.

- Patients' user experience of 'TelePraCMan' is measured by the User Experience Questionnaire (UEQ [36]).

- Identification of general support needs and/or potential persons giving support in dealing with 'TelePraCMan' or technical problems. This selfadministered questionnaire is a 9-items instrument that measures what kind of support (e.g. family members, manual, or without support) is used in case of technical problems and how often it used. All items rated with a 7-point Likert scale with response options from 'never' to 'always'.

- Sociodemographic data.

Secondary outcomes concerning GP-practice side (GPs and VERAHs) are:

- The general technical affinity of participating GPs and VERAHs measured with the TA-EG questionnaire [35].

- Perception and acceptance of 'TelePraCMan' by GPs and VERAHs and the compatibility with GP-practices workflows are measured with a selfadministered 21-items questionnaire at baseline (T0) and a 30-items questionnaire at follow-up (T1). All items rated with a 5-point Likert scale with response options from 'disagree' to 'strongly agree'. Furthermore, there is a field for 'no statement' for each item. The follow-up questionnaire (T1) offers several fields for open answers.

- Sociodemographic data.

After finalizing the 6-months intervention period, a qualitative process evaluation consisting of semi-structured interviews with GPs, VERAHs and patients is planned to investigate the experiences made with 'TelePraCMan' during practical use.

Table 1 shows the enrolment, and the points of outcomes assessment. 


\begin{tabular}{|c|c|c|c|c|}
\hline & \multicolumn{4}{|c|}{ STUDY PERIOD } \\
\hline & Enrolment & Allocation & $\begin{array}{c}\text { Post- } \\
\text { allocation }\end{array}$ & Close-out \\
\hline TIMEPOINT & -TO & $\begin{array}{l}0 \text { month } \\
\text { (TO) }\end{array}$ & $\begin{array}{l}6 \text { months } \\
\text { (T1) }\end{array}$ & 7 Months \\
\hline \multicolumn{5}{|l|}{ ENROLMENT: } \\
\hline \multirow{3}{*}{$\begin{array}{r}\text { Eligibility screen } \\
\text { Informed consent } \\
\text { Allocation }\end{array}$} & $\mathrm{x}$ & & & \\
\hline & $x$ & & & \\
\hline & & $x$ & & \\
\hline \multicolumn{5}{|l|}{ INTERVENTION: } \\
\hline Provision of ,TelePraCMan & & $\bullet$ & $\longrightarrow$ & \\
\hline \multicolumn{5}{|l|}{ ASSESSMENTS (PATIENTS): } \\
\hline $\mathrm{SF}-12$ & & $\mathrm{x}$ & $\mathrm{x}$ & \\
\hline PAM13-D & & $x$ & $x$ & \\
\hline TA-EG & & $\mathrm{x}$ & & \\
\hline Smartphone usage & & $\mathrm{x}$ & & \\
\hline Log-In data & & & $x$ & \\
\hline UEQ & & & $x$ & \\
\hline $\begin{array}{r}\text { Support with technical problems } \\
\text { Socialdemographic data }\end{array}$ & & $\mathrm{x}$ & $x$ & \\
\hline Socialdemographic data & & $\mathrm{x}$ & & \\
\hline Semi-structured Interview & & & & $x$ \\
\hline \multicolumn{5}{|l|}{$\begin{array}{l}\text { ASSESSMENTS (GPS AND } \\
\text { VERAHs): }\end{array}$} \\
\hline TA-EG & & $x$ & & \\
\hline Perception and acceptance & & $\mathrm{x}$ & $x$ & \\
\hline Sociodemographic data & & $\mathrm{x}$ & $\mathrm{x}$ & \\
\hline Semi-structured Interview & & & & $\mathrm{x}$ \\
\hline
\end{tabular}

\section{Sample size}

In order to be able to calculate a meaningful sample size for the study, recommendations refer to include at least 50 participants per arm to achieve a power of $80 \%$ and an effect size of $d<0.1$ (2-side significance level of 5\%) [37]. For the randomized trial we assumed a small effect size because studies measuring health-related quality of life with SF-12 with comparable populations showed no significant effects after four month [18] or showed significant effects at the earliest after 24 month [29]. Since the randomized study in this case has a duration of only six months, a lower effect would suffice as an indication of the effectiveness of 'TelePraCMan'. Because the heterogenous combination of chronically diseases by multimorbid probands and a drop-out rate up to $26 \%$ in studies with comparable interventions [16,38],[19] we operate with a total of 100 participants per arm in order to be able to consider a spectrum of chronically disease combinations as broad as possible in a later subgroup analyses. A comparable intervention study in a similar population and setting shows an intracluster correlation (ICC) of 0,01 at practice level [39]. This minimal effect has no noticeable influence on the power, so that we do not explicitly calculate the design effect $D[40]$. In conclusion, a total of 100 patients per arm is targeted.

\section{Recruitment}

To reach the sample size, hundred selected GP-practices participating in 'PraCMan' will be informed by letter and/or fax about the study and will be asked for their support in patient recruitment and study conduction. Practices size and geographical closeness are primary selection criteria. The aim is to recruit a maximum of 20 GP-practices with a sufficient number of 'PraCMan' patients from all over the federal state of Baden-Wuerttemberg.

The recruitment of the participating patients is carried out by the GP-practices. Patients who meet the inclusion criteria will be addressed by the participating GP-practices and will be informed verbally about the aim and content of the study by the VERAH. If the patient is interested to participate in the study, he receives two sets of information material - one for the intervention group and one for the control group. After reading the information material, the patient can formally agree to participate in the study, knowing that due to the randomization process he might end up in either in the intervention or in the control group.

Before study enrolment, practice teams will receive a short training imparting knowledge on the use of 'TelePraCMan' as well as on the recruitment and randomization processes.

\section{Interviews}

Interviews with GPs, VERAHs and patients will be conducted after completing the 6-months intervention period, to evaluate the implementation strategy. For this, patients participating as part of the intervention group receive a letter from their GP-practice containing the T1-questionaire as well as an invitation to participate in an interview dealing with their 'TelePraCMan'-experiences. Patients interested in interview participation are asked to directly contact the study central office.

Furthermore, participating GPs and VERAHs will be recruited for semi-structured interviews after the first patient the practice recruited finishes the 6-months intervention period.

Patients' and practice team members' interview participation will be financially rewarded.

\section{Randomization}


Randomization of the patients is executed by practice staff on patient level. After the patients give consent to study participation, they will be handed out sealed envelopes with the corresponding group membership. The sealed envelopes will be prepared by the study central office beforehand ensuring that their content cannot be inspected by the GP-practices until they are opened by the patients. For the randomization, patients are randomly assigned to either the intervention or the control group by lot. This procedure is constructed to result in equally sized groups. Blocks of ten Patient-IDs will be individually matched with five control-group and five intervention-group notes.

Because the intervention cannot be blinded, all study members are able to know the group assignment of a patient from the moment of opening the envelope on. This is necessary to perform all further processes in this study as guarding the patient within the installation process as well as comparing data of the two study groups.

\section{Data collection and management}

\section{Questionnaire}

Paper-based questionnaires for participating patients at baseline (T0) and follow-up (T1) are stored in the Investigator Site File (ISF) in the GP-practices. With recruitment and allocation, the patients receive the baseline questionnaire (T0) and a post-paid envelope. The completed questionnaires will be sent by mail to the study central office by patients. After six months, the GP-practice will be given the follow-up questionnaire (T1) and a post-paid envelope to the patient, who sends the filled in questionnaire by mail to the study central office, too.

The paper-based questionnaires for participating GPs and VERAHs at baseline (T0) and a post-paid envelope are handed out to GP-practice teams at the enrolment in study face-to-face or by mail by researchers of the study central office. With the mail for recruitment to the interviews for the process evaluation, the GP-practice teams receive the follow-up questionnaire (T1) and a post-paid envelope, to send the completed questionnaire via mail to the study central office. Paper-based documents will be stored in the study central office at the Department of General Practice and Health Services Research at University Hospital Heidelberg.

\section{Interviews}

Semi-structured interviews will be conducted as face-to-face or telephone interviews by researchers of the study central office. The estimated duration for the interviews will be a maximum duration of one hour. All interviews will be audio-recorded, transcribed verbatim and stored on servers of the University Hospital Heidelberg. The transcripts of interviews will contain pseudonymized data only.

\section{Data analysis}

Data will be analyzed in accordance with the CONSORT-EHealth V1.6.2. statement [41]. Intention-to-treat analysis will be performed in the primary analysis. Descriptive statistics will be used to summarize characteristics of staff of GP-practices and participants by group. The primary and secondary outcomes at follow-up will be compared between study arms, adjusted for baseline values and potential confounders in a multiple regression analysis. To explore the impact of contextual factors (differences between participating practices, sociodemographic data of the patients such as age and number of chronic conditions, group affiliation, timepoint of data collection (T0 vs. T1), etc.), we will apply a multilevel regression that considers the hierarchical structure of data [40]. If needed, comparisons of specific variables on outcome level between the control and the intervention group and the two collection time points (T0 and T1) will be conducted using t-test or a non-parametric testing equivalent depending on the individual characteristics of the variables such as the scale level. Quantitative data analysis will be conducted using the statistical programs R, Excel and SPSS Version 25.

Additional sensitive analyses will be conducted as per-protocol analysis in the intervention group (i.e. regular users of the intervention).

Missing data of multi-item scales will be handled according to questionnaire guidelines. In case of missing data in SF-12 no score can be calculated due the weighting of the questions [42]. If no guideline is available for the other questionnaires, then items will be imputed with the Expectation-MaximizationAlgorithm.

The qualitative data collected via semi-structured interviews at close-out will be fully transcribed and a deductive thematic content analysis [43] will be conducted, guided by the checklist for 'Tailored Implementation for Chronic Diseases' (TICD) [44] using the framework method [45.

\section{Discussion}

Usage of 'TelePraCMan' is expected to contribute to improve the self-management of multimorbid patients and, thus, to reinforce the feeling of control over one's own well-being. The acceptance and interest by patients as well as GP-practices regarding mHealth-interventions in the GP-care setting is expected to be a critical factor. Especially among older, multimorbid persons, technologies such as smartphones or tablets are not fully established, although there is a positive trend [46]. If the results show positive effects, then 'TelePraCMan' will be adjusted for further use and research.

The only comparable study showed no effects on health-related quality of life [18], and problems with integration in GP-practices workflow [19]. Nevertheless, in supplementary interviews the patients still reported increased self-confidence and motivation as a result of the intervention [18].

Therefore, a small effect on the health-related quality of life can be expected for our study. However, the fact that 'TelePraCMan' builds up on the already established GP-practice based care-management 'PraCMan' could lead to better integration into the practice's workflow and a measurable higher quality of life for participating patients. 


\section{Strengths and Limitations}

Given the characteristics of the intervention, the participating patients and the treating GP-practices know which patients are in the intervention group or control group and cannot be blinded for the intervention. Therefore, bias due to deviations from the intended intervention might be possible. Furthermore, allocation procedures are constructed adequately to prevent concealment bias. Since 'TelePraCMan' is a supplement to 'PraCMan' and not a stand-alone intervention, the results may not be easily transferred to other settings. Generalizability is also influenced by the expectedly increased inclusion of patients who show a positive attitude towards mHealth interventions. Because we defined the possession of a smartphone or tablet as an inclusion criterion, we also expect these patients to have a higher technical affinity. To capture this impact, we chose specific items and questionnaires with whom we can measure these affinities and attitudes and include the observed effects in our outcomes evaluation.

'TelePraCMan' is one of the first mHealth-interventions explicitly designed for multimorbid patients. If it gets accepted by GP-practices' staff and patients and proves as effective, 'TelePraCMan' in combination with 'PraCMan' will contribute to benefit patients' individual treatment plan. Furthermore, potential health complications could possibly be identified earlier. An advantage of the study is the relatively equal care of patients in the 'PraCMan' care context. This limits the influence of heterogeneous medical care on the results. From a scientific perspective, the results of the study can contribute to a better understanding of the acceptance and effectiveness of mHealth-interventions among multimorbid patients and elderly people.

If the conduction of the pilot study results in a measured and perceived benefit of the application for practice teams and patients, an expansion of TelePraCMan to make it available for all PraCMan patients in Germany is planned.

\section{Abbreviations}

COPD: chronic obstructive pulmonary disease; GP: General practitioners; GP-practice: General practitioner practice; ISF: Investigator Site File; PRAM 13-D: German version of the "Patient Activation Measure" questionnaire; TA-EG: questionnaire to general technical affinity; TICD: Tailored Implementation for Chronic Diseases; UEQ: User Experience Questionnaire; VERAH: Care assistant in GP-practice;

\section{Declarations}

\section{Ethics approval and consent to participate}

The study protocol has been submitted to and approved by the ethics committee of the Medical Faculty Heidelberg (S-092/2019) as well as the ethics committee of the State Medical Council of Baden-Wuerttemberg (B-F-2019-073) prior to the start of the study.

All study participants give their written consent for participation. In case of the conducted field study, participants gave their consent by submitting the questionnaire.

\section{Consent for publication}

Not applicable

\section{Availability of data and materials}

Data sharing is not applicable to this article as no datasets were generated or analyzed during the preparation of this study protocol.

\section{Competing interests}

Dr. Freund and Dr. Szecsenyi report about a patent with royalties paid for the PraCMan Cockpit software. The authors not named here declare that they have no competing interests.

\section{Funding}

This study is funded by the Federal Ministry of Social Affairs and Integration of Baden-Wuerttemberg, Germany. The funder had no role in the design of the study and will not be involved in its execution, data analysis and interpretation of results. Neither was the funder involved in writing this manuscript.

\section{Authors' contributions}

Study concept and design involved LW, AW, NL, TF and JS. NL, LW and AW drafted the original manuscript. Critical revision of the manuscript for important intellectual content involved JS, TF, AM and MW.

Acknowledgements: For their important work and/or support in the conduction of the study we want to thank our colleagues Annika Baldauf, Marion Kiel, Anja Schumann and Frank Aluttis. Furthermore, we would like to sincerely thank Linda Göbl and Alexander Villhauer for their important input.

\section{References}

1. Lehnert T, König H-H. Auswirkungen von Multimorbidität auf die Inanspruchnahme medizinischer Versorgungsleistungen und die Versorgungskosten. Bundesgesundheitsblatt - Gesundheitsforschung - Gesundheitsschutz. 2012;55:685-92. doi:10.1007/s00103-012-1475-6. 
2. Laux G, Kuehlein T, Rosemann T, Szecsenyi J. Co- and multimorbidity patterns in primary care based on episodes of care: results from the German CONTENT project. BMC Health Services Research. 2008;8:14. doi:10.1186/1472-6963-8-14.

3. Dodel R. Multimorbidität: Konzept, Epidemiologie, Versorgung. Der Nervenarzt. 2014;85:401-8. doi:10.1007/s00115-013-3937-y.

4. Mino-León D, Reyes-Morales H, Doubova SV, Pérez-Cuevas R, Giraldo-Rodríguez L, Agudelo-Botero M. Multimorbidity Patterns in Older Adults: An Approach to the Complex Interrelationships Among Chronic Diseases. Arch Med Res. 2017;48:121-7. doi:10.1016/j.arcmed.2017.03.001.

5. Fuchs J, Busch M, Lange C, Scheidt-Nave C. Prevalence and patterns of morbidity among adults in Germany. Bundesgesundheitsblatt Gesundheitsforschung - Gesundheitsschutz. 2012;55:576-86. doi:10.1007/s00103-012-1464-9.

6. van den Bussche H. Die Zukunftsprobleme der hausärztlichen Versorgung in Deutschland: Aktuelle Trends und notwendige Maßnahmen. Bundesgesundheitsblatt - Gesundheitsforschung - Gesundheitsschutz. 2019;62:1129-37. doi:10.1007/s00103-019-02997-9.

7. Freund T, Peters-Klimm F, Rochon J, Mahler C, Gensichen J, Erler A, et al. Primary care practice-based care management for chronically ill patients (PraCMan): study protocol for a cluster randomized controlled trial [ISRCTN56104508]. Trials. 2011a;12:163. doi:10.1186/1745-6215-12-163.

8. Llor C, Moragas A, Miravitlles M. Usefulness of a patient symptom diary card in the monitoring of exacerbations of chronic bronchitis and chronic obstructive pulmonary disease. Int J Clin Pract. 2012;66:711-7. doi:10.1111/j.1742-1241.2012.02930.x.

9. Eastwood CA, Travis L, Morgenstern TT, Donaho EK. Weight and symptom diary for self-monitoring in heart failure clinic patients. J Cardiovasc Nurs. 2007;22:382-9. doi:10.1097/01.JCN.0000287027.49628.a7.

10. Peters-Klimm F, Olbort R, Campbell S, Mahler C, Miksch A, Baldauf A, Szecsenyi J. Physicians' view of primary care-based case management for patients with heart failure: a qualitative study. Int J Qual Health Care. 2009;21:363-71. doi:10.1093/intqhc/mzp032.

11. Kenning C, Fisher L, Bee P, Bower P, Coventry P. Primary care practitioner and patient understanding of the concepts of multimorbidity and selfmanagement: A qualitative study. SAGE Open Med. 2013;1:2050312113510001. doi:10.1177/2050312113510001.

12. Hodek JM, Ruhe A, Greiner W. Gesundheitsbezogene Lebensqualität bei Multimorbidität im Alter. Bundesgesundheitsblatt - Gesundheitsforschung Gesundheitsschutz. 2009;52:1188. doi:10.1007/s00103-009-0974-6.

13. Kitsiou S, Paré G, Jaana M, Gerber B. Effectiveness of mHealth interventions for patients with diabetes: An overview of systematic reviews. PLOS ONE. 2017;12(3):e0173160.

14. Steele Gray C, Wodchis WP, Upshur R, Cott C, McKinstry B, Mercer S, et al. Supporting Goal-Oriented Primary Health Care for Seniors with Complex Care Needs Using Mobile Technology: Evaluation and Implementation of the Health System Performance Research Network, Bridgepoint Electronic Patient Reported Outcome Tool. JMIR Res Protoc. 2016;5:e126. doi:10.2196/resprot.5756.

15. Steele Gray C, Miller D, Kuluski K, Cott C. Tying eHealth Tools to Patient Needs: Exploring the Use of eHealth for Community-Dwelling Patients With Complex Chronic Disease and Disability. JMIR Res Protoc. 2014;3:e67. doi:10.2196/resprot.3500.

16. Steele Gray C, Khan Al, Kuluski K, McKillop I, Sharpe S, Bierman AS, et al. Improving Patient Experience and Primary Care Quality for Patients With Complex Chronic Disease Using the Electronic Patient-Reported Outcomes Tool: Adopting Qualitative Methods Into a User-Centered Design Approach. JMIR Res Protoc. 2016;5:e28. doi:10.2196/resprot.5204.

17. Irfan Khan A, Gill A, Cott C, Hans PK, Steele Gray C. mHealth Tools for the Self-Management of Patients With Multimorbidity in Primary Care Settings: Pilot Study to Explore User Experience. JMIR Mhealth Uhealth. 2018;6:e171. doi:10.2196/mhealth.8593.

18. Steele Gray C, Gravesande J, Hans PK, Nie JX, Sharpe S, Loganathan M, et al. Using Exploratory Trials to Identify Relevant Contexts and Mechanisms in Complex Electronic Health Interventions: Evaluating the Electronic Patient-Reported Outcome Tool. JMIR Form Res. 2019;3:e11950. doi:10.2196/11950.

19. Steele Gray C, Gill A, Khan Al, Hans PK, Kuluski K, Cott C. The Electronic Patient Reported Outcome Tool: Testing Usability and Feasibility of a Mobile App and Portal to Support Care for Patients With Complex Chronic Disease and Disability in Primary Care Settings. JMIR Mhealth Uhealth. 2016;4:e58. doi:10.2196/mhealth.5331.

20. Scheibe M, Reichelt J, Bellmann M, Kirch W. Acceptance factors of mobile apps for diabetes by patients aged 50 or older: a qualitative study. Med 20. 2015;4:e1. doi:10.2196/med20.3912.

21. Sill J, Steenbock B, Helmer S, Zeeb H, Pischke CR. Apps zur Förderung von körperlicher Aktivität - Nutzung und Einstellungen bei Erwachsenen im Alter von 50 Jahren und älter. Prävention und Gesundheitsförderung 2018. doi:10.1007/s11553-018-0674-x.

22. Steinert A, Haesner M, Tetley A, Steinhagen-Thiessen E. Nutzungsverhalten einer Gesundheitsapplikation zum Selbstmonitoring von Senioren. Prävention und Gesundheitsförderung. 2015;10:281-6. doi:10.1007/s11553-015-0510-5.

23. Steinert A, Haesner M, Steinhagen-Thiessen E. App-basiertes Selbstmonitoring bei Typ-2-Diabetes. Zeitschrift für Gerontologie und Geriatrie. 2017;50:51623. doi:10.1007/s00391-016-1082-5.

24. Noël PH, Frueh BC, Larme AC, Pugh JA. Collaborative care needs and preferences of primary care patients with multimorbidity. Health Expect. 2005;8:5463. doi:10.1111/j.1369-7625.2004.00312.x.

25. Scheidt-Nave C, Richter S, Fuchs J, Kuhlmey A. Herausforderungen an die Gesundheitsforschung für eine alternde Gesellschaft am Beispiel „Multimorbidität". Bundesgesundheitsblatt - Gesundheitsforschung - Gesundheitsschutz. 2010;53:441-50. doi:10.1007/s00103-010-1052-9.

26. Chan A-W, Tetzlaff JM, Gøtzsche PC, Altman DG, Mann H, Berlin JA, et al. SPIRIT 2013 explanation and elaboration: guidance for protocols of clinical trials. BMJ. 2013;346:e7586. doi:10.1136/bmj.e7586.

27. Diederichs C, Berger K, Bartels DB. The measurement of multiple chronic diseases-a systematic review on existing multimorbidity indices. J Gerontol A Biol Sci Med Sci. 2011;66(3):301-11.

28. Johnston MC, Crilly M, Black C, Prescott GJ, Mercer SW. Defining and measuring multimorbidity: a systematic review of systematic reviews. European Loading [MathJax]/jax/output/CommonHTML/jax.js 2-9. 
29. Freund T, Peters-Klimm F, Boyd CM, Mahler C, Gensichen J, Erler A, et al. Medical Assistant-Based Care Management for High-Risk Patients in Small Primary Care Practices: A Cluster Randomized Clinical Trial. Ann Intern Med. 2016;164:323-30. doi:10.7326/M14-2403.

30. Lentferink AJ, Oldenhuis HK, Groot M de, Polstra L, Velthuijsen H, van Gemert-Pijnen JE. Key Components in eHealth Interventions Combining SelfTracking and Persuasive eCoaching to Promote a Healthier Lifestyle: A Scoping Review. Journal of medical Internet research. 2017;19:e277. doi:10.2196/jmir.7288.

31. Morfeld M, Kirchberger I, Bullinger M. SF-36: Fragebogen zum Gesundheitszustand. Göttingen: Hogrefe; 2011.

32. Brenk-Franz K, Hibbard JH, Herrmann WJ, Freund T, Szecsenyi J, Djalali S, et al. Validation of the German version of the patient activation measure 13 (PAM13-D) in an international multicentre study of primary care patients. PLoS ONE. 2013;8:e74786. doi:10.1371/journal.pone.0074786.

33. Greene J, Hibbard JH. Why does patient activation matter? An examination of the relationships between patient activation and health-related outcomes. J Gen Intern Med. 2012;27:520-6. doi:10.1007/s11606-011-1931-2.

34. Yadav UN, Lloyd J, Hosseinzadeh H, Baral KP, Bhatta N, Harris MF. Self-management practice, associated factors and its relationship with health literacy and patient activation among multi-morbid COPD patients from rural Nepal. BMC Public Health. 2020;20:300. doi:10.1186/s12889-020-8404-7.

35. Karrer K, Glaser C, Clemens C, Bruder C. Technikaffınität erfassen - der Fragebogen TA-EG. ZMMS Spektrum. 2009:196-201.

36. Laugwitz B, Schrepp M, Held T. Konstruktion eines Fragebogens zur Messung der User Experience von Softwareprodukten. In: Heinecke HM, Paul H, editors. Mensch und Computer 2006. München: OLDENBOURG WISSENSCHAFTSVERLAG; 2006. doi:10.1524/9783486841749.125.

37. Whitehead AL, Julious SA, Cooper CL, Campbell MJ. Estimating the sample size for a pilot randomised trial to minimise the overall trial sample size for the external pilot and main trial for a continuous outcome variable. Stat Methods Med Res. 2016;25:1057-73. doi:10.1177/0962280215588241.

38. Whitehead L, Seaton P. The Effectiveness of Self-Management Mobile Phone and Tablet Apps in Long-term Condition Management: A Systematic Review. Journal of medical Internet research. 2016;18:e97-e97. doi:10.2196/jmir.4883.

39. Peters-Klimm F, Campbell S, Hermann K, Kunz CU, Müller-Tasch T, Szecsenyi J. Case management for patients with chronic systolic heart failure in primary care: the HICMan exploratory randomised controlled trial. Trials. 2010;11:56. doi:10.1186/1745-6215-11-56.

40. Hox JJ. Multilevel analysis: Techniques and applications. 2nd ed. New York, NY: Routledge; 2010.

41. Eysenbach G. CONSORT-EHEALTH: improving and standardizing evaluation reports of Web-based and mobile health interventions. J Med Internet Res. 2011;13:e126. doi:10.2196/jmir.1923.

42. Morfeld M, Dietsche S, Bürger W, Koch U. Der SF-12 - Das Problem der Missing Data. Diagnostica. 2003;49:129-35. doi:10.1026//0012-1924.49.3.129.

43. Mayring P. Qualitative Inhaltsanalyse: Grundlagen und Techniken. 12th ed. Weinheim, Basel: Beltz Verlag; 2015.

44. Flottorp SA, Oxman AD, Krause J, Musila NR, Wensing M, Godycki-Cwirko M, et al. A checklist for identifying determinants of practice: a systematic review and synthesis of frameworks and taxonomies of factors that prevent or enable improvements in healthcare professional practice. Implement Sci.

2013;8:35. doi:10.1186/1748-5908-8-35.

45. Gale NK, Heath G, Cameron E, Rashid S, Redwood S. Using the framework method for the analysis of qualitative data in multi-disciplinary health research. BMC Med Res Methodol. 2013;13:117. doi:10.1186/1471-2288-13-117.

46. eMarketer. Smartphones Are All the Rage in Germany. 24.6.2014. https://www.emarketer.com/Article/Smartphones-All-Rage-Germany/1010942. Accessed 30 Nov 2018.

\section{Figures}

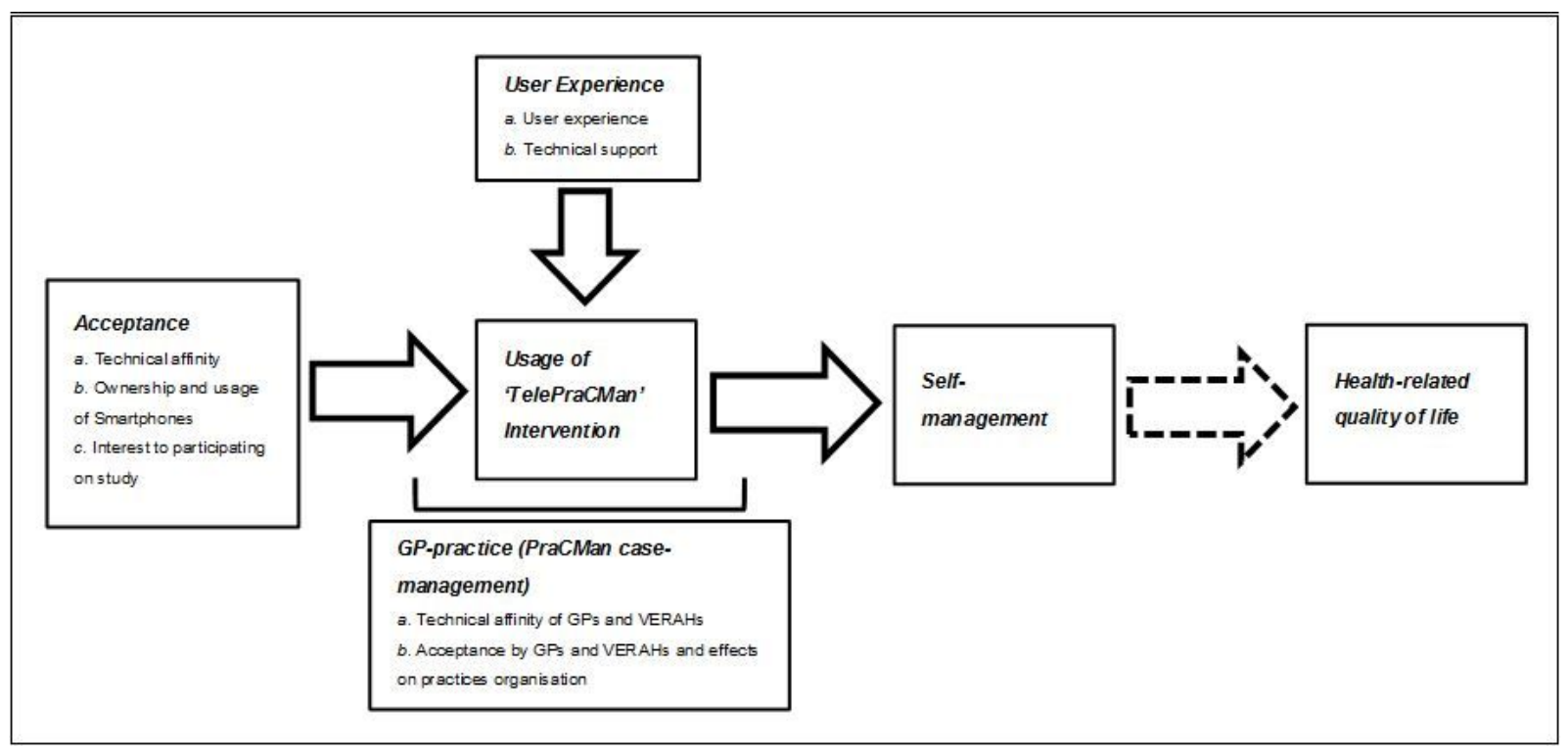

Figure 1 


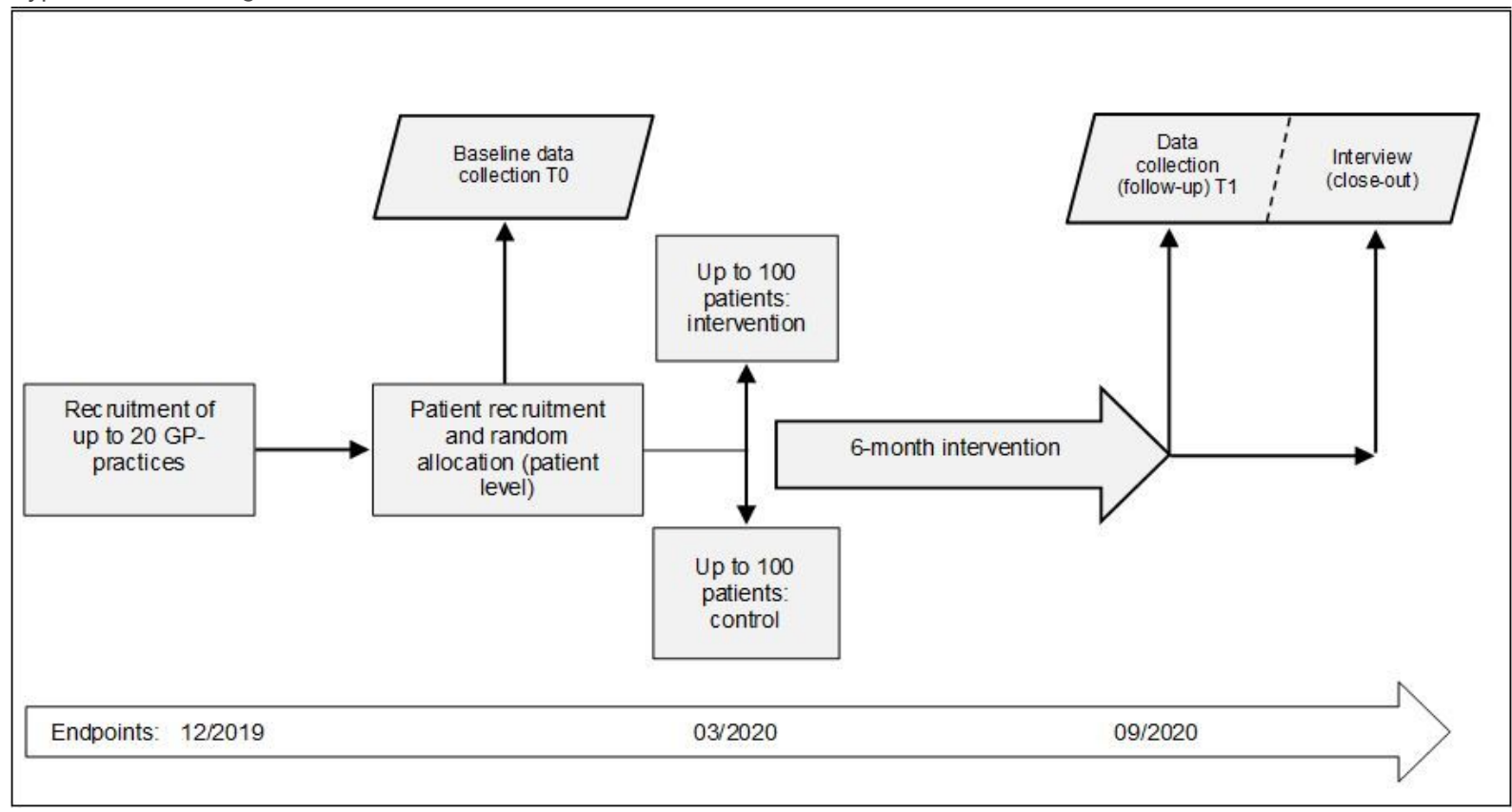

Figure 2

Design and timeframe of the TelePraCMan trail

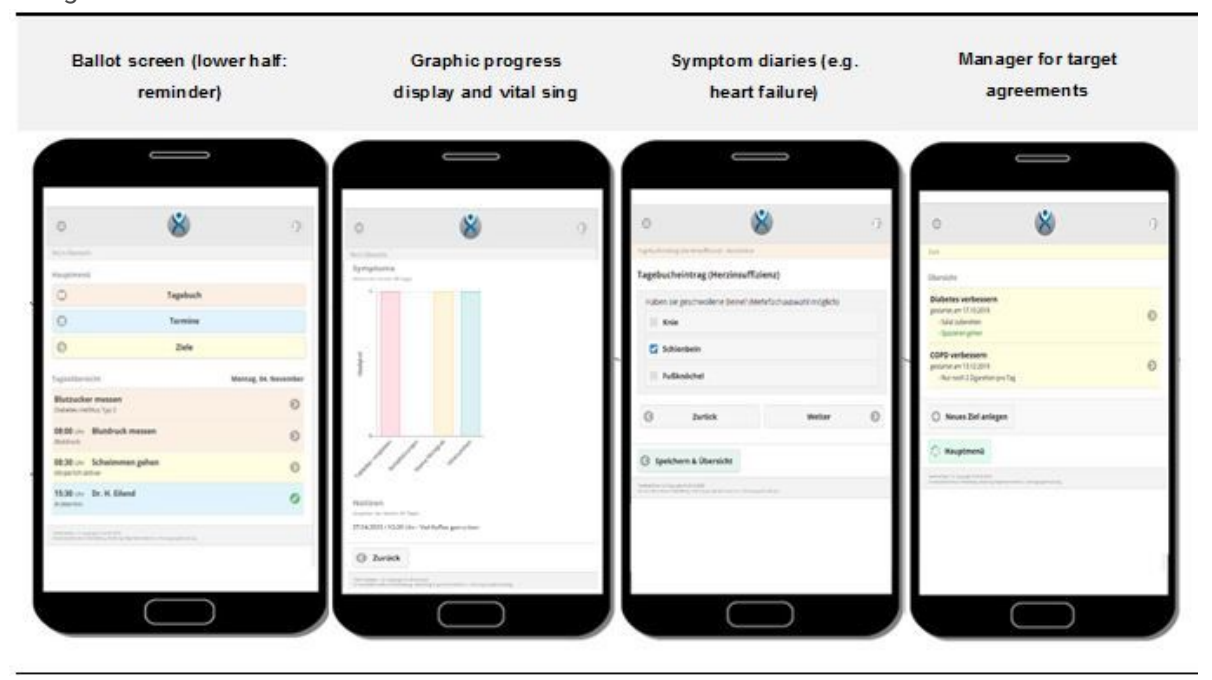

Figure 3

Main features of 'TelePraCMan'. The wallpaper of the smartphones was created by Bartekhdd and was downloaded from https://pixabay.com/de/vectors/smartphone-symbol-moderne-schwarz-1557796/, Accessed 12. May 2020. 